6. Mostafa HH, Hardick J, Morehead E, Miller JA, Gaydos CA, Manabe YC. Comparison of the analytical sensitivity of seven commonly used commercial SARS-CoV-2 automated molecular assays. J Clin Virol 2020; 130: 104578 .

7. Rakotosamimanana N, Randrianirina F, Randremanana R, et al. GeneXpert for the diagnosis of COVID-19 in LMICs. Lancet Glob Health 2020; 8: e1457-8.

DOI: https://doi.org/10.1016/j.pathol.2021.10.001

\section{Analytical sensitivity and specificity of the Cepheid Xpert Xpress SARS-CoV-2/FIu/RSV assay}

Sir,

The COVID-19 pandemic caused by SARS-CoV-2 is a public health emergency on a global scale, with over 85 million cases worldwide as of 6 January 2021. Along with clear and decisive public health interventions, one of the cornerstones in controlling the pandemic is rapid and accurate diagnostic testing, with reverse transcription polymerase chain reaction (RT-PCR) testing considered the 'gold standard' testing method. ${ }^{2}$ The clinical spectrum of COVID-19 is broad, with an overlap between COVID-19 clinical features and symptoms of other common respiratory viral infections such as influenza and respiratory syncytial virus (RSV). ${ }^{3}$ Given the importance of early detection of cases of COVID-19, rapid discrimination between SARS-CoV-2 and other respiratory viruses is essential.

The Xpert Xpress SARS-CoV-2 assay (Cepheid, USA) has been used in many countries for the rapid detection of SARSCoV-2, with high sensitivity and specificity, ${ }^{4,5}$ including 89 remote point of care testing sites across Australia. ${ }^{6}$ The assay detects both the pan-sarbecovirus $\mathrm{E}$ gene and the $\mathrm{N} 2$ region of the $\mathrm{N}$ gene specific to SARS-CoV-2 in approximately 45 minutes. ${ }^{4}$ Recently, the Cepheid Xpert Xpress SARS-CoV-2/ Flu/RSV assay has received Emergency Use Authorization (EUA) from the United States Food and Drug Administration (FDA). ${ }^{7}$ It is designed to detect and differentiate SARS-CoV2 , influenza A, influenza B and RSV in nasopharyngeal swabs, nasal swabs or nasal washes/aspirates and is used on GeneXpert Systems. Analytical results are available within 36 minutes and provide a single detected or not detected result for each virus type and a matching $\mathrm{Ct}$ value result for interpretation.

Here, we undertook a clinical and laboratory validation study to evaluate the analytical sensitivity and specificity of the Xpert Xpress SARS-CoV-2/Flu/RSV assay.

Testing was conducted at the Microbiological Diagnostic Unit Public Health Laboratory (MDU PHL), The University of Melbourne at the Doherty Institute, Melbourne, Australia. In brief, SARS-CoV-2-positive nasopharyngeal or deep nasal swabs were obtained from routine clinical testing at MDU PHL, and stored SARS-CoV-2-negative nasopharyngeal or deep nasal swabs were obtained from the Department of Microbiology, Royal Melbourne Hospital (RMH), Melbourne, Australia.

All clinical samples were previously tested for SARSCoV-2 using the AusDiagnostics Coronavirus Typing (8well) panel (AusDiagnostics, Australia) at RMH, as previously described, ${ }^{8}$ and using the Aptima SARS-CoV-2 assay
(Hologic, USA) at MDU PHL, according to the manufacturer's instructions.

Analytical sensitivity for SARS-CoV-2 detection was assessed using two approaches. First, a $50 \mu \mathrm{L}$ volume of quantified inactivated whole virus (SARS-CoV-2 Analytical Q Panel; Qnostics, UK) supplied as a standardised dilution series $(6.0-1.7 \log 10$ digital copies/mL) was spiked into universal transport media (UTM). Subsequently, $300 \mu \mathrm{L}$ of spiked UTM was used in the Xpert Xpress SARS-CoV-2/Flu/RSV assay and also in the Xpert Xpress SARS-CoV-2 assay for comparison. The limit of detection (LOD) was determined, and all testing was performed in triplicate. Second, analytical sensitivity was also determined using heat-killed SARS-CoV-2 virus stock quantified at $1.04 \times 10^{5} \mathrm{TCID}_{50} / \mathrm{mL}$, obtained from previously isolated SARS-CoV-2 in Melbourne.? Virus was diluted in saline, and $50 \mu \mathrm{L}$ was spiked into universal transport media (obtained from the Media Preparation Unit, University of Melbourne). Three replicates at $10 \times, 1 \times$ and $0.1 \times$ LOD (LOD determined by Xpert Xpress SARS-CoV2 ) were tested. Clinical sensitivity was assessed by testing 46 RT-PCR confirmed positive samples (previously tested on the Panther Fusion SARS-CoV-2 assay; Hologic), spanning a range of cycle $\mathrm{Ct}$ values between 18.2 and 36.1 (Table 1).

Cross-reactivity was assessed using a commercial panel of respiratory control organisms (NATRPC2-BIO; Zeptometrix, USA) comprising purified, intact virus particles and bacterial cells suspended in a matrix that mimics the composition of a clinical specimen. Cross-reactivity was also examined using gamma-irradiated influenza virus (A/Victoria/31/2020 and B/ Darwin/58/2019), respiratory syncytial virus (RSV) A 16144363 and RSV B 15136810 and two seasonal human coronavirus strains OC43 and 229E obtained from the Victorian Infectious Diseases Reference Laboratory (VIDRL), Melbourne, Australia, and spiked into pooled nasopharyngeal swab samples that tested negative to SARSCoV-2. Clinical specificity was assessed by testing 50 SARSCoV-2 RT-PCR-negative samples obtained from patients with respiratory symptoms attending $\mathrm{RMH}$.

Using the Qnostics SARS-CoV-2 panel, the LoD for SARS-CoV-2 with the Xpert Xpress SARS-CoV-2/Flu/RSV assay was 8.3 copies $/ \mathrm{mL}$, and for the Xpert Xpress SARSCoV-2, assay was 8.3 copies $/ \mathrm{mL}$. Using dilutions of heat inactivated SARS-CoV-2, the LoD for the Xpert Xpress SARS-CoV-2/Flu/RSV assay was 0.002 TCID $_{50} / \mathrm{mL}$, and for the Xpert Xpress, SARS-CoV-2 assay was 0.002 TCID $_{50}$ /

Table 1 Sensitivity and specificity of the Xpert Xpress SARS-CoV-2/Flu/ RSV test with the comparator SARS-CoV-2 assay (using the Aptima SARSCoV-2 assay)

\begin{tabular}{lccc}
\hline GeneXpert Ct & GeneXpert $^{\mathrm{a}}$ & RT-PCR $^{\mathrm{b}}$ & Concordance (\%) $^{\text {a }}$ \\
\hline Positive samples & & & \\
$\quad<20$ & 21 & 21 & 100 \\
$20-25$ & 10 & 10 & 100 \\
$26-30$ & 9 & 6 & 100 \\
$>30$ & 6 & 50 & 100 \\
Negative samples & 50 & & 100 \\
$\quad$ N/A & &
\end{tabular}

${ }^{\mathrm{a}}$ Tested on the Xpert Xpress SARS-CoV-2/Flu/RSV assay.

${ }^{\mathrm{b}}$ Tested on the Aptima SARS-CoV-2 assay. 
Table 2 Evaluation of limit of detection of the Xpert Xpress SARS-CoV-2/Flu/RSV assay using the Qnostics SARS-CoV-2 Analytical Q Panel and dilutions of heat-inactivated virus

\begin{tabular}{|c|c|c|c|c|}
\hline \multirow[t]{2}{*}{ Virus/Strain } & \multicolumn{2}{|c|}{ Xpert Xpress SARS-CoV-2/Flu/RSV assay } & \multicolumn{2}{|c|}{ Xpert SARS-CoV-2 assay } \\
\hline & Limit of detection & Ct value ${ }^{a}$ & Limit of detection & Ct value ${ }^{a}$ \\
\hline \multicolumn{5}{|l|}{ Qnostics panel } \\
\hline SARS-CoV-2 & 8.33 copies $/ \mathrm{mL}$ & 39.6 & 8.33 copies $/ \mathrm{mL}$ & $\begin{array}{l}\text { N2 region } 40.4 \\
\text { E gene } 39.9\end{array}$ \\
\hline \multicolumn{5}{|l|}{ Inactivated virus } \\
\hline SARS-CoV-2 (VIC01) & $0.002 \mathrm{TCID} 50 / \mathrm{mL}$ & 30.3 & $0.002 \mathrm{TCID} 50 / \mathrm{mL}$ & $\begin{array}{l}\text { N2 region } 41.0 \\
\text { E gene } 33.8\end{array}$ \\
\hline Influenza A/Vic/31/2020 & $0.042 \mathrm{TCID} 50 / \mathrm{ml}$ & 37.5 & - & - \\
\hline Influenza B/Darwin/58 2019 & $0.0004 \mathrm{TCID} 50 / \mathrm{ml}$ & 37.6 & - & - \\
\hline RSV A 16144363 & $0.043 \mathrm{TCID} 50 / \mathrm{ml}$ & 34.2 & - & - \\
\hline RSV B 15136810 & $0.022 \mathrm{TCID} 50 / \mathrm{ml}$ & 34.9 & - & - \\
\hline
\end{tabular}

${ }^{\text {a }}$ Cycle threshold, mean of three replicates.

$\mathrm{mL}$. Agreement of the Xpert Xpress SARS-CoV-2/Flu/RSV assay with the Panther Fusion SARS-CoV-2 assay was $100 \%$, across a range of $\mathrm{Ct}$ values (Table 1). Inactivated influenza A B and RSV were serially diluted in UTM; $10 \mu \mathrm{L}$, $25 \mu \mathrm{L}$ and $50 \mu \mathrm{L}$ of diluted virus were spiked into pooled negative swab matrix at approx $1 \times, 2 \times$ and $5 \times$ LOD (previously determined by MDU). Further limiting dilutions were prepared from the above spike preparations and diluted in negative swab matrix to determine LOD and were tested in triplicate. The LoD values for influenza and RSV strains are described in Table 2.

Using the Zeptometrix respiratory panel, all expected targets were detected using the Xpert Xpress SARS-CoV-2/Flu/
RSV assay. In addition, no cross-reactivity was observed across any of the four assay channels in the Xpert Xpress SARS-CoV-2/Flu/RSV assay (SARS-CoV-2; influenza A; influenza B; RSV). Further, the Xpert Xpress SARS-CoV-2 assay did not display any cross-reactivity with the Zeptometrix panels.

Using heat-inactivated virus spiked into negative swab samples, no cross-reactivity was observed, and all expected positive samples were detected (Table 3).

Moreover, all 50 clinical samples that tested negative for SARS-CoV-2 at RMH also tested negative using the Xpert Xpress SARS-CoV-2/Flu/RSV assay, giving a negative percentage agreement of $100 \%$.

Table 3 Cross-reactivity and specificity across reactivity testing of the Xpert Xpress SARS-CoV-2/Flu/RSV

\begin{tabular}{|c|c|c|c|c|c|}
\hline \multirow[t]{2}{*}{ Panel/Virus } & \multicolumn{4}{|c|}{ Xpert Xpress SARS-CoV-2/Flu/RSV } & \multirow[t]{2}{*}{ Xpert SARS-CoV-2 assay } \\
\hline & SARS-CoV-2 & Influenza A & Influenza B & RSV & \\
\hline \multicolumn{6}{|c|}{ NATRPC2-BIO, Zeptometrix, pool 1} \\
\hline Adenovirus Type 1 & - & + & - & - & - \\
\hline \multicolumn{6}{|l|}{ Adenovirus Type 3} \\
\hline \multicolumn{6}{|l|}{ Adenovirus Type 31} \\
\hline \multicolumn{6}{|c|}{ C. pneumoniae (CWL-029) } \\
\hline \multicolumn{6}{|c|}{ Influenza A 2009 H1N1 (A/NY/02/2009) } \\
\hline \multicolumn{6}{|c|}{ Influenza A H3N2 (A/Brisbane/10/07) } \\
\hline \multicolumn{6}{|c|}{ Human metapneumovirus Type 8 (Peru6-2003) } \\
\hline \multicolumn{6}{|c|}{ M. pneumoniae (M129) } \\
\hline \multicolumn{6}{|l|}{ Parainfluenza Type 1} \\
\hline \multicolumn{6}{|l|}{ Parainfluenza Type 4} \\
\hline \multicolumn{6}{|c|}{ Rhinovirus Type $1 \mathrm{~A}$} \\
\hline \multicolumn{6}{|c|}{ NATRPC2-BIO, Zeptometrix, pool 2} \\
\hline B. parapertussis (A747) & - & + & + & + & - \\
\hline \multicolumn{6}{|c|}{ Coronavirus HKU-1 recombinant } \\
\hline \multicolumn{6}{|c|}{ Coronavirus (NL63) } \\
\hline \multicolumn{6}{|c|}{ Coronavirus (OC43) } \\
\hline \multicolumn{6}{|c|}{ Influenza A H1N1 (A/New Cal/20/99) } \\
\hline \multicolumn{6}{|c|}{ Influenza B (B/Florida/02/06) } \\
\hline \multicolumn{6}{|l|}{ Parainfluenza Type 2} \\
\hline \multicolumn{6}{|l|}{ Parainfluenza Type 3} \\
\hline \multicolumn{6}{|l|}{ RSV A (2006 isolate) } \\
\hline \multicolumn{6}{|l|}{ Inactivated virus } \\
\hline SARS-CoV-2 (VIC01) & + & - & - & - & + \\
\hline Coronavirus OC43 & - & - & - & - & - \\
\hline Coronavirus 229E & - & - & - & - & - \\
\hline Influenza A/Vic/31/2020 & - & + & - & - & - \\
\hline Influenza B/Darwin/58 2019 & - & - & + & - & - \\
\hline RSV A 16144363 & - & - & - & + & - \\
\hline
\end{tabular}


Here, we provide a comprehensive evaluation of the performance of the new Xpert Xpress SARS-CoV-2/Flu/ RSV assay. We demonstrate that the performance characteristics of this assay are comparable to the existing Xpert Xpress SARS-CoV-2 assay for the detection of SARS-CoV2, with similar LoD and specificity for both assays. Further, our results are in keeping with a recent study by Mostafa et al. that demonstrated an overall positive percentage agreement for the SARS-CoV-2 target of $98.7 \%$ when compared to a range of other RT-PCR platforms (including the Xpert Xpress SARS-CoV-2 and Hologic Panther Fusion SARS-CoV-2 assays), and a negative percentage agreement of $100 \%$ with other targets (influenza A/B and RSV) showing $100 \%$ total agreement. ${ }^{6}$ More recently, Lueng et al. also reported high concordance between the Xpert Xpress SARS-CoV-2 and Xpert Xpress SARS-CoV-2/Flu/RSV assay. ${ }^{10}$ For end-users, one notable difference exists between the two Xpert assay types. The single Xpert Xpress SARS-CoV-2 assay reports N2 and E cycle threshold values independently within the analyte results for each sample. The test also calls out three result types: positive, presumptive positive and not detected. In contrast, the Xpert Xpress SARS-CoV-2/Flu/RSV assay reports a single $\mathrm{Ct}$ value for each of the four pathogens and each result is reported as detected or undetected. While some purchase cost differential is likely between the single Xpert SARS-CoV-2 assay and its 4 plex cousin, commercial pricing had not been finalised at the time of writing.

More broadly, rapid and reliable testing for SARS-CoV-2 has been critical to the COVID-19 response. Given the overlap in clinical symptoms between COVID-19 and other respiratory illnesses such as influenza, rapid differentiation of causative pathogens is essential in ensuring appropriate clinical and public health control measures. This is particularly important for responses to COVID-19, i.e., rapid isolation, treatment intervention and contact tracing. Moreover, as COVID-19 vaccination is gradually implemented globally, and national and international movement resumes over the next few years, inevitably, the circulation of other respiratory viruses such as influenza and RSV will increase.

There is both an immediate and ongoing need for rapid multiplex testing, particularly testing that can be performed near or at the point of care, such as the Xpert Xpress SARSCoV-2/Flu/RSV assay which requires minimal sample preparation steps. Use of this assay in combination with a validated viral inactivating transport medium ${ }^{11}$ also reduces pathogenic exposure risk for test operators at that point of care or in laboratory settings. Possible uses include testing in aged care and emergency hospital settings, where rapid diagnosis will enable triage of patients to appropriate treatment, isolation rooms, and prompt implementation of infection control measures. Remote or low resource locations could also benefit from either Xpert COVID molecular point of care test given they may reduce the need for laboratory reflex testing and shorten clinical decision-making time. In addition, the presence of such a test could avert unnecessary isolation and costly evacuation of some individuals to larger health facilities.
Acknowledgements: Our sincere thanks to Tanya Applegate, Socheata Chea and Eloise Williams for their support with this evaluation.

Conflicts of interest and sources of funding: Laboratory work was supported by a grant from the NHMRC Medical Research Future Fund (APP2002317). We acknowledge inkind support from Cepheid who supplied the SARS-CoV-2/ Flu A-B/RSV cartridges free of charge for this validation study.

\section{Nicole Isles ${ }^{1, *}$, Steven G. Badman ${ }^{2, *}$, Susan Ballard ${ }^{1}$, Bowen Zhang ${ }^{3,4}$, Benjamin P. Howden ${ }^{1,4}$, Rebecca Guy ${ }^{2, *}$, Deborah A. Williamson ${ }^{1,3,4,}$}

${ }^{1}$ Microbiological Diagnostic Unit Public Health Laboratory, The University of Melbourne at the Peter Doherty Institute for Infection and Immunity, Melbourne, Vic, Australia; ${ }^{2}$ Kirby Institute, University of New South Wales, Sydney, NSW, Australia; ${ }^{3}$ Department of Microbiology, Royal Melbourne Hospital, Melbourne, Vic, Australia; ${ }^{4}$ Department of Microbiology and Immunology, The University of Melbourne at the Peter Doherty Institute for Infection and Immunity, Melbourne, Vic, Australia; "these authors contributed equally

Contact Dr Steven G. Badman.

E-mail: sbadman@kirby.unsw.edu.au

1. World Health Organization. WHO Coronavirus Disease (COVID-19) Dashboard. Cited 10 Jan 2021. https://covid19.who.int

2. Kretzschmar ME, Rozhnova G, Bootsma MCJ, van Boven M, van de Wijgert J, Bonten MJM. Impact of delays on effectiveness of contact tracing strategies for COVID-19: a modelling study. Lancet Public Health 2020; 5: e452-9.

3. Huang C, Wang Y, Li X, et al. Clinical features of patients infected with 2019 novel coronavirus in Wuhan, China. Lancet 2020; 395: 497-506.

4. Loeffelholz MJ, Alland D, Butler-Wu SM, et al. Multicenter evaluation of the Cepheid Xpert xpress SARS-CoV-2 test. J Clin Microbiol 2020; 58: e00926-20.

5. Wolters F, van de Bovenkamp J, van den Bosch B, et al. Multi-center evaluation of Cepheid Xpert ${ }^{\circledR}$ Xpress SARS-CoV-2 point-of-care test during the SARS-CoV-2 pandemic. J Clin Virol 2020; 128: 104426.

6. Hengel B, Causer L, Matthews S, et al. A decentralised point-of-care testing model to address inequities in the COVID-19 response. Lancet Infect Dis 2021; 21: e183-90.

7. Mostafa HH, Carroll KC, Hicken R, et al. Multi-center evaluation of the Cepheid Xpert ${ }^{\circledR}$ xpress SARS-CoV-2/flu/RSV test. J Clin Microbiol 2021; 59: e02955-20.

8. Caly L, Druce J, Roberts J, et al. Isolation and rapid sharing of the 2019 novel coronavirus (SARS-CoV-2) from the first patient diagnosed with COVID-19 in Australia. Med J Aust 2020; 212: 459-62.

9. Williams $\mathrm{E}$, Bond $\mathrm{K}$, Chong $\mathrm{B}$, et al. Implementation and evaluation of a novel real-time multiplex assay for SARS-CoV-2: in-field learnings from a clinical microbiology laboratory. Pathology 2020; 52 754-9.

10. Leung EC, Chow VC, Lee MK, Tang KP, Li DK, Lai RW. Evaluation of the Xpert Xpress SARS-CoV-2/Flu/RSV assay for simultaneous detection of SARS-CoV-2, Influenza A/B and respiratory syncytial viruses in nasopharyngeal specimens. J Clin Microbiol 2021; 59: e02965-20.

11. van Bockel D, Munier CML, Turville S, et al. Evaluation of commercially available viral transport medium (VTM) for SARS-CoV-2 inactivation and use in point-of-care (POC) testing. Viruses 2020; 12: 1208.

DOI: https://doi.org/10.1016/j.pathol.2021.09.002 